\title{
Fibro - odontoma ameloblástico; reporte de caso clínico
}

\author{
Graciela Susana Martínez, ${ }^{1}$ (i) Analía Budkin, ${ }^{2}$ (i) Úrsula Anaya Flores. ${ }^{2}$ (i)
}

\begin{abstract}
Resumen: El fibro - odontoma ameloblástico es un tumor odontogénico benigno con células epiteliales odontogénicas cúbicas, tejido conjuntivo fibroso embrionario y epitelio odontogénico primitivo. Se presenta con poca frecuencia y afecta principalmente a niños y adultos jóvenes entre la primera y segunda década de vida. Este trabajo es un reporte del caso clínico de un paciente de sexo masculino de 12 años de edad atendido en el Hospital de Odontología Infantil "Don Benito Quinquela Martín", al cual se le diagnostica un tumor odontogénico benigno asociado a un canino permanente inferior derecho impactado. Se realiza un plan de tratamiento integral, individualizado y con fuerte componente preventivo, trabajando siempre en constante interrelación con los servicios de Odontología Preventiva, Cirugía, Radiología y Ortodoncia del hospital. En una primera etapa se tratan las infecciones prevalentes y se realiza el refuerzo del huésped. Posteriormente se realiza la intervención quirúrgica para extirpar la lesión y las estructuras dentarias asociadas a esta. La evolución del paciente fue favorable permitiendo continuar su tratamiento en el servicio de Ortodoncia. Objetivo: Describir la situación clínica de un paciente masculino de 12 años de edad, con diagnóstico de fibro - odontoma ameloblástico con un enfoque interdisciplinario y dentro de un plan de tratamiento integral. Conclusión: El tratamiento realizado con un enfoque interdisciplinario y dentro de un plan integral, permitió lograr un resultado favorable en el abordaje del fibro - odontoma ameloblástico.
\end{abstract}

Palabras clave: Diagnóstico precoz, fibroodontoma ameloblástico, cirugía, diente impactado.

\section{Fibro - odontoma ameloblástico; relato de caso clínico}

Resumo: O fibro - odontoma ameloblástico é um tumor odontogênico benigno com células epiteliais odontogênicas cuboides, tecido conjuntivo fibroso embrionário e epitélio odontogênico primitivo. Se apresenta com pouca frequência e afeta principalmente crianças e adultos jovens entre a primeira e segunda década de vida. Este trabalho é um relato de caso clínico de um paciente de sexo masculino de 12 anos de idade atendido no Hospital de Odontologia Infantil "Don Benito Quinquela Martín"; diagnosticado com um tumor odontogênico benigno associado a um canino permanente inferior direito impactado. É realizado um plano de tratamento integral, individualizado e com forte componente preventivo, trabalhando sempre em constante inter-relação entre os Serviços de Odontologia Preventiva, Cirurgia, Radiologia e Ortodontia do hospital. Numa primeira fase, são tratadas as infecções prevalentes e é realizado o reforço do hospedeiro. Posteriormente é realizada a intervenção cirúrgica para remover a lesão e as estruturas dentárias associadas a ela. A evolução do paciente foi favorável, permitindo que ele continue o tratamento no Serviço de Ortodontia. Objetivo: Descrever a situação clínica de um paciente masculino de 12 anos de idade, com diagnóstico de fibro - odontoma ameloblástico com uma abordagem interdisciplinar e dentro de um plano de tratamento integral. Conclusão: $O$ tratamento realizado com uma abordagem interdisciplinar e dentro de um plano integral, permitiu alcançar um resultado favorável na abordagem do fibro - odontoma ameloblástico.

Palabra-chave: Diagnóstico precoce, fibro - odontoma ameloblástico, cirurgia, dente impactado.

\footnotetext{
Especialista en Odontopediatría. Jefa de Unidad del Servicio de Odontología Preventiva. Hospital de Odontología Infantil "Don Benito Quinquela Martín", Ciudad Autónoma de Buenos Aires, Argentina.

2 Especialista en Odontopediatría. Odontóloga de Planta del Servicio de Odontología Preventiva. Hospital de Odontología Infantil "Don Benito Quinquela Martín", Ciudad Autónoma de Buenos Aires, Argentina.
} 


\section{Ameloblastic fibro odontoma; clinical case report}

Abstract: Ameloblastic fibro-odontoma is an infrequent benign odontogenic tumor with cubic odontogenic epithelia cells, embryonic fibrous connective tissue, and primitive odontogenic epithelium. It mainly affects children and young adults between the first and second decade of life. This article is a clinical case report of a 12 - year - old male patient seen at Hospital of Pediatric Dentistry "Don Benito Quinquela Martín," where he was diagnosed with a benign odontogenic tumor associated with an impacted permanent lower right canine tooth. The treatment plan included a multidisciplinary collaboration among the Departments of Preventive Dentistry, Surgery, Radiology, and Orthodontics. In the first stage, prevalent infections were treated and the host was strengthened. The second stage was the surgica removal of the lesion and associated dental structures. The patient's course was favorable and continued his treatment at the Department of Orthodontics. Objective: To describe the clinical course of a 12-year-old male patient diagnosed with ameloblastic fibro-odontoma managed with an interdisciplinary approach in the setting of a comprehensive treatment plan. Conclusion: An interdisciplinary management in the setting of a comprehensive plan resulted in a favorable approach to ameloblastic fibro-odontoma.

Key words: Early diagnosis, ameloblastic fibro odontoma, surgery, impacted tooth

\section{Introducción}

Según la clasificación de la Organización Mundial de la Salud de los tumores odontogénicos publicada en el año
2005, el fibro - odontoma ameloblástico

(FOA) pertenece al grupo de lesiones con epitelio odontogénico que contiene ectomesénquima odontogénico con o sin formación de tejido duro dentario (Tabla 1). ${ }^{1}$

Tabla 1: Clasificación histológica de los tumores odontogénicos benignos (OMS, 2005).

\begin{tabular}{|l|}
\hline 1. Epitelio odontogénico con estroma fibroso maduro \\
\hline sin ectomesénquima odontogénico \\
\hline Ameloblastoma sólido/multiquístico \\
\hline Ameloblastoma extraóseo/tipo periférico \\
\hline Ameloblastoma tipo desmoplástico \\
\hline Ameloblastoma tipo uniquístico \\
\hline Tumor odontogénico escamoso \\
\hline Tumor odontogénico epitelial calcificante \\
\hline Tumor odontogénico adenomatoide \\
\hline Tumor odontogénico queratoquístico \\
\hline
\end{tabular}

3. Mesénquima y/o ectomesénquima odontogénico con o sin epitelio odontogénico

Fibroma odontogénico

Mixoma odontogénico (mixofibroma)

Cementoblastoma

\begin{tabular}{l} 
2. Epitelio odontogénico con ectomesénquima \\
odontogénico, con o sin formación de tejido dentario duro \\
\hline Fibroma ameloblástico \\
\hline Fibrodentinoma ameloblástico \\
\hline Fibroodontoma ameloblástico \\
\hline Odontoma \\
\hline Odontoma, tipo complejo \\
Odontoma, tipo compuesto \\
Odontoameloblastoma \\
\hline Tumor odontogénico quístico calcificante \\
\hline Tumor dentinogénico de células fantasmas \\
\hline
\end{tabular}

4.Lesiones relacionadas al hueso

Fibroma osificante

Displasia fibrosa

Displasias óseas

Lesión central de células gigantes(granuloma)

Querubismo

Quiste óseo aneurismático

Quiste óseo simple

Quiste óseo simple


El mismo, es semejante al fibroma ameloblástico, el cual muestra cambios inductivos que permiten la formación en diversos grados de una matriz de esmalte y dentina. ${ }^{1}$ Algunos autores sostienen que el fibroma ameloblástico y el odontoma complejo parecen estar combinados en esta lesión. ${ }^{2}$

El FOA es un tumor odontogénico benigno que se presenta con poca frecuencia. ${ }^{3} \mathrm{La}$ etiología del FOA es aún desconocida y controversial, ya que imita el desarrollo del germen dentario durante el período embrionario pero de forma aberrante, evidenciando diversos grados de interacciones inductivas entre el epitelio y el ectomesénquima odontogénico, los cuales se traducen en la formación de esmalte y dentina dentro del tejido mesodérmico celular que se asemeja a la papila dental. ${ }^{4}$

Se encuentra bien circunscripto, ubicado generalmente en la porción posterior del maxilar o la mandíbula, de crecimiento lento, que expande corticales y tiende a producir aumento de volumen. En la mayoría de los casos se asocia a dientes no erupcionados. ${ }^{5,6}$ Por lo general cursa asintomático, por lo que el hallazgo de este tipo de lesiones es durante revisiones radiográficas de rutina. ${ }^{7}$ EI FOA afecta principalmente a niños y adultos jóvenes entre la primera y segunda década de vida, con una media de edad de 14 años. ${ }^{8}$ No muestra predilección por género, aunque hay autores que sostienen una leve tendencia hacia el género masculino. ${ }^{9}$

Radiográficamente corresponde a una lesión radiolúcida uni o multilocular bien delimitada, con cantidades variables de contenido radiopaco en su interior que se asemejan a tejido dentario en diferentes etapas de la odontogénesis. Además, puede presentar expansión de las tablas corticales, por lo que es difícil diferenciarlo de otras lesiones de aspecto similar, como el ameloblastoma o el quiste dentígero cuando está asociado a un diente retenido. ${ }^{10}$

Histológicamente es una lesión encapsulada constituida por hebras y cordones delgados de células epiteliales odontogénicas cúbicas, tejido conjuntivo fibroso embrionarioyepitelio odontogénico primitivo, simulando el retículo estrellado. ${ }^{11}$

El tratamiento del FOA se asocia con un enfoque quirúrgico conservador. Las recurrencias esporádicas del mismo se han atribuido a la extracción quirúrgica inadecuada en el momento del tratamiento inicial. Algunos otros informes demostraron que la enucleación conservadora es suficiente. $^{12}$ Existe controversia en la literatura con respecto a la extracción o retención del germen del diente asociado en el caso del FOA. La mayoría de los artículos afirman que el germen del diente asociado debe eliminarse para evitar la recurrencia. ${ }^{6,13,14}$

El propósito de este reporte es describir la situación clínica de un paciente masculino de 12 años de edad, con diagnóstico de fibro - odontoma ameloblástico con un enfoque interdisciplinario y dentro de un plan de tratamiento integral.

\section{Relato de caso}

Paciente de sexo masculino de 12 años de edad, sin antecedentes médicos personales 
ni familiares de interés, concurre al Hospital de Odontología Infantil "Don Benito Quinquela Martín", siendo el motivo de la consulta: "caries". El mismo presenta experiencia odontológica previa en otra institución. Ingresa para su atención en el servicio de Odontología Preventiva.

Al examen clínico intraoral se observa persistencia de canino temporario inferior derecho (8.3), ausencia de canino permanente inferior derecho (4.3) y abombamiento de tabla ósea vestibular en zona de dicho canino, sin sintomatología dolorosa espontánea ni tampoco a la palpación (Figuras 1 y 2).

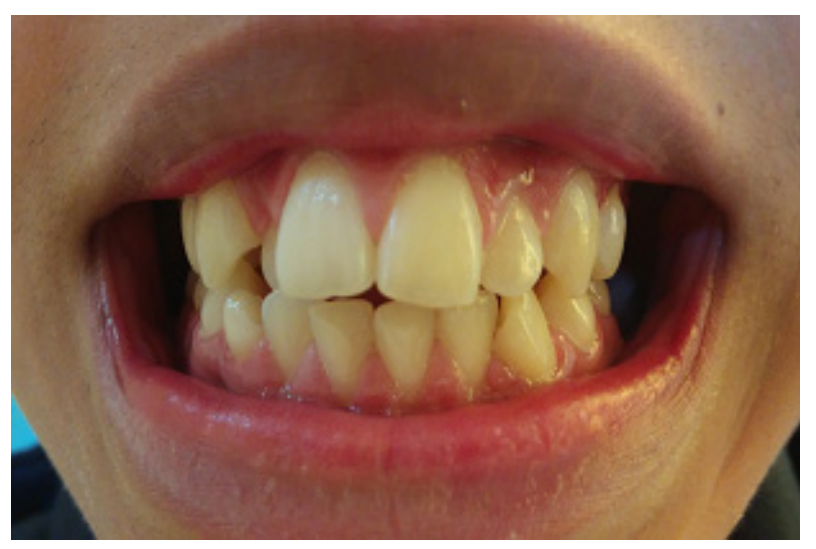

Figura 1. Aspecto clínico intrabucal. Se observa persistencia de la pieza 8.3.

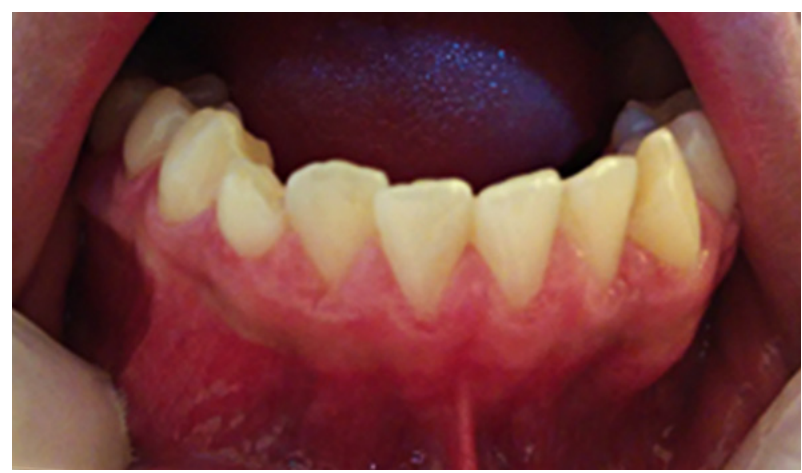

Figura 2. Abombamiento de la tabla ósea vestibular en zona de la pieza 4.3.
Al examen radiográfico con radiografías panorámica y periapical (Figuras 3 y 4), se observa: pieza 4.3 retenida cuya corona se encuentra rodeada por imagen radiolúcida de más de $2 \mathrm{~mm}$ de diámetro y presencia de imágenes radiopacas compatibles con dentículos.

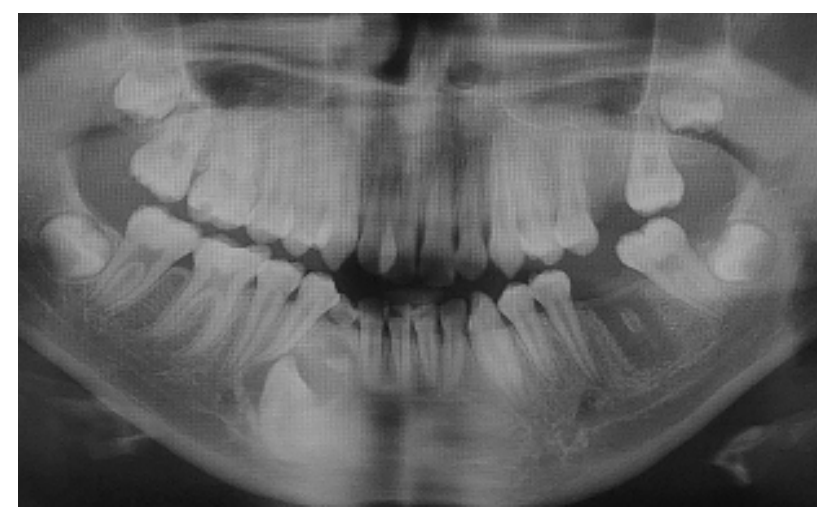

Figura 3. Radiografía panorámica prequirúrgica. Se observa persistencia de pieza 8.3, imagen compatible con odontoma y pieza 4.3 retenida.

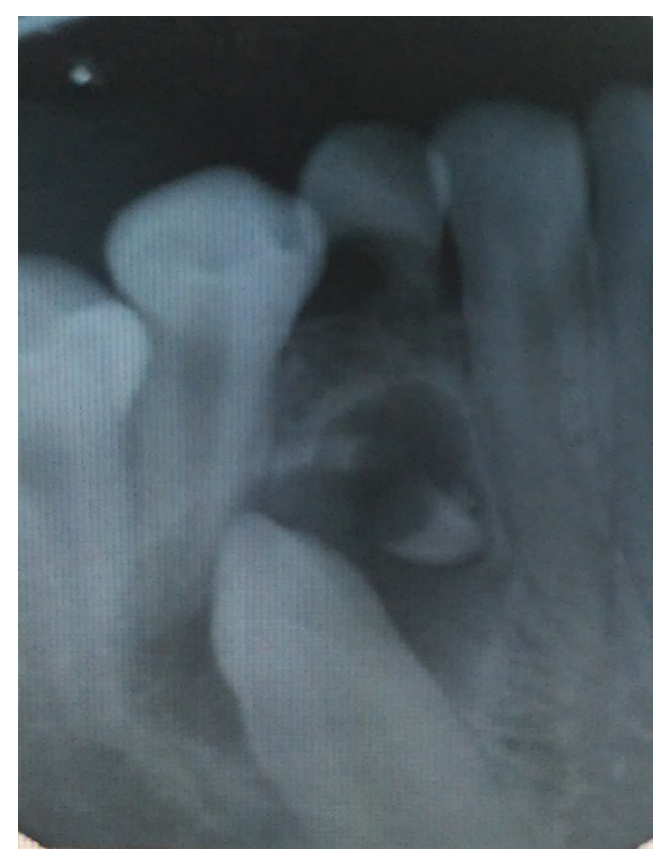

Figura 4. Radiografía periapical prequirúrgica 
Se realiza un plan de tratamiento integral, individualizado y con fuerte componente preventivo, el cual incluye acciones preventivas y terapéuticas en función del riesgo individual, que permitirán elaborar un diagnóstico correcto. La evaluación integral deberá incluir: estado general y crecimiento, tejidos blandos extrabucales e intrabucales, higiene y salud gingivoperiodontal, tejidos duros intrabucales, desarrollo de la oclusión y conducta del niño. ${ }^{15}$

Una vez realizadas las explicaciones pertinentes a los padres, estos firmaron el correspondiente consentimiento informado establecido por el Gobierno de la Ciudad Autónoma de Buenos Aires - Ministerio de Salud (Resolución 0356/ MSGC/09 - Ley 153 - decretos N²08/01 y $\left.\mathrm{N}^{\circ} 2316 / 03\right)$ para la realización del tratamiento propuesto, así como también para el registro, documentación del caso y eventual publicación posterior.
En simultáneo con la realización del plan de tratamiento integral, individualizado y con fuerte componente preventivo se realizan interconsultas desde el servicio de Odontología Preventiva con los servicios de Cirugía y Ortodoncia del hospital.

En el servicio de Cirugía se completa el examen radiográfico de la zona con técnica de Clark (Figura 5). En esta se observa que al distalar el cono, la lesión se desplaza hacia mesial en dirección contraria, por lo que se considera que la misma se encuentra para su abordaje quirúrgico por la zona vestibular.

En conjunto con el servicio de Ortodoncia, se realiza el estudio respectivo de la oclusión y se considera la posición desfavorable de la pieza 4.3 y la posibilidad de recurrencia de la lesión. Se decide realizar las exodoncias de las piezas 8.3 y 4.3 retenida y eliminación de la lesión.
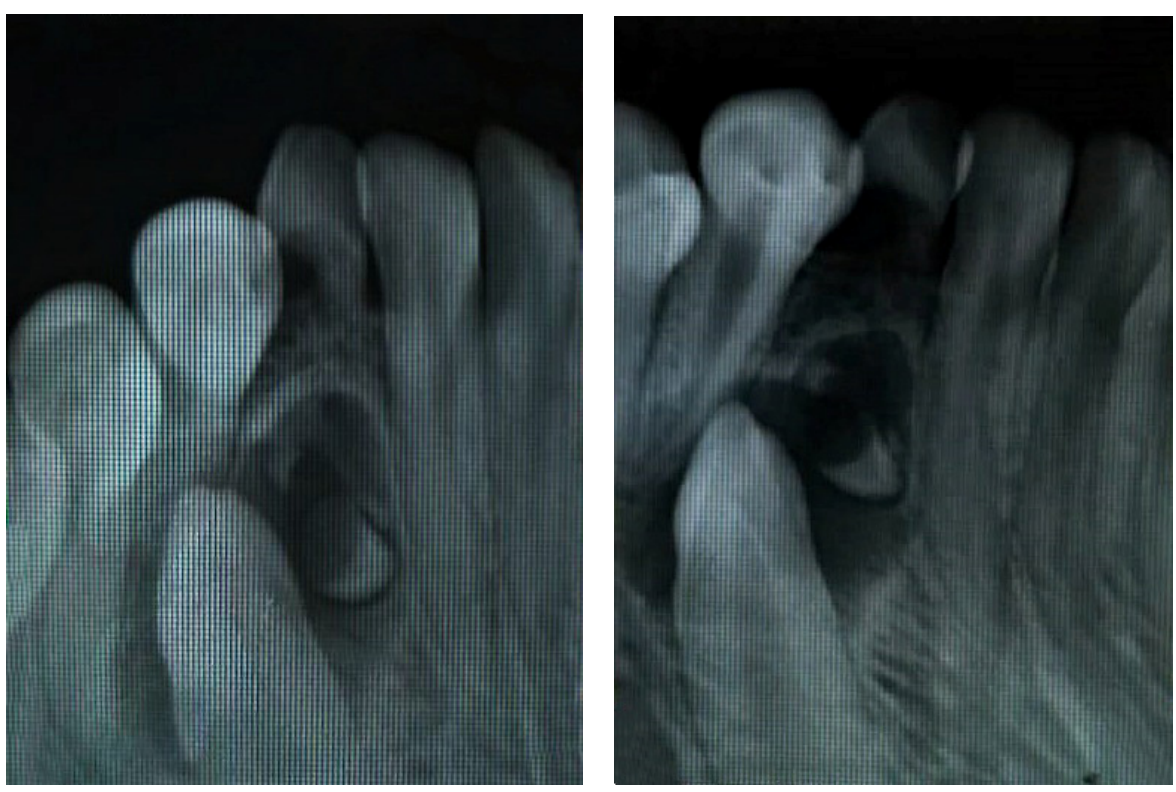

Figura 5. Técnica de Clark. 
Se completa la consulta prequirúrgica con análisis clínicos de laboratorio y, teniendo en cuenta que el paciente presenta experiencia en el servicio de Cirugía del hospital por exodoncias de las piezas 2.6 y 3.6, se refuerza la motivación y se realizan las explicaciones pertinentes al grupo familiar acerca del tipo de procedimiento a realizar, con el objeto de favorecer la colaboración y el éxito de la intervención.

\section{Técnica quirúrgica}

Se realiza antisepsia intrabucal con clorhexidina $0.12 \%$ y bajo anestesia local troncular e infiltrativa con clorhidrato de carticaína 4\% - adrenalina 1:100.000, se levanta un colgajo mucoperióstico. Se procede a las exodoncias del canino temporario inferior derecho (8.3) y canino permanente inferior derecho retenido (4.3) mediante la técnica de odontosección como recurso quirúrgico (Figuras 6 y 7). Para estas maniobras se utilizó: forceps de incisivo inferior, elevador recto y fresa cilíndrica para turbina.

Se remueve el saco pericononario de

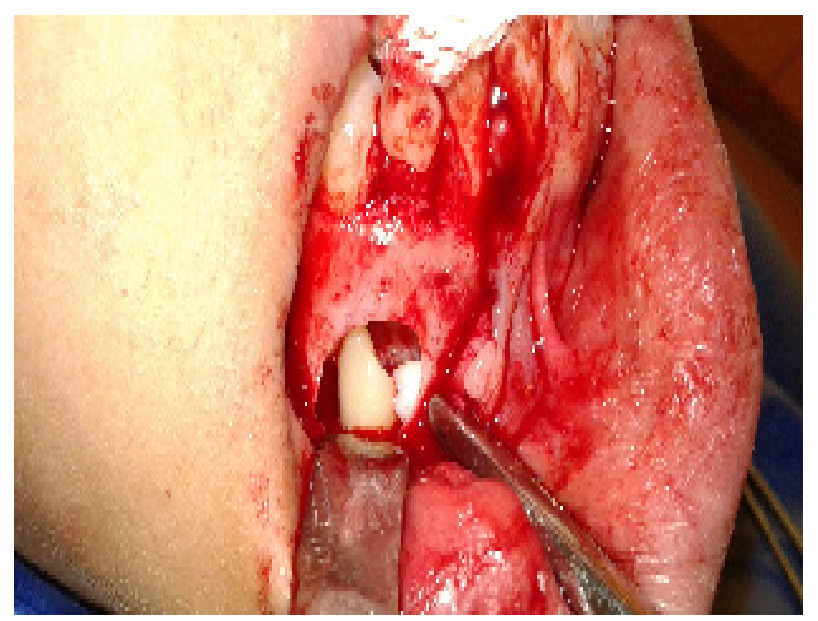

Figura 6. Imagen intraoperatoria. Se observa pieza 4.3 retenida y fibro - odontoma ameloblástico.

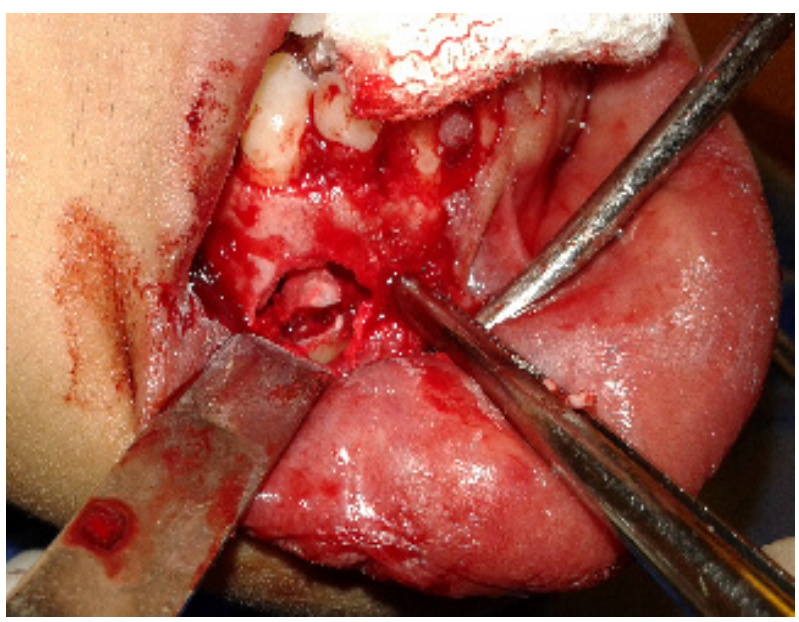

Figura 7. Odontosección de la pieza 4.3.

aspecto quístico de la pieza 4.3 y de los dentículos observados radiográficamente, completando la intervención con la toma de una radiografía periapical intraquirúrgica para corroborar la extirpación total de los mismos y se sutura con hilo de origen sintético no reabsorbible (Figuras $8 \mathrm{~A}, 8 \mathrm{~B}$ y $8 C) .{ }^{8}$ Se indica al paciente medicación antibiótica (amoxicilina $500 \mathrm{mg}$ ) y analgésica (ibuprofeno $400 \mathrm{mg}$ ) por vía oral y buches nocturnos de clorhexidina 0,12 \% una vez por día durante una semana.

La muestra de tejidos duros (hueso y

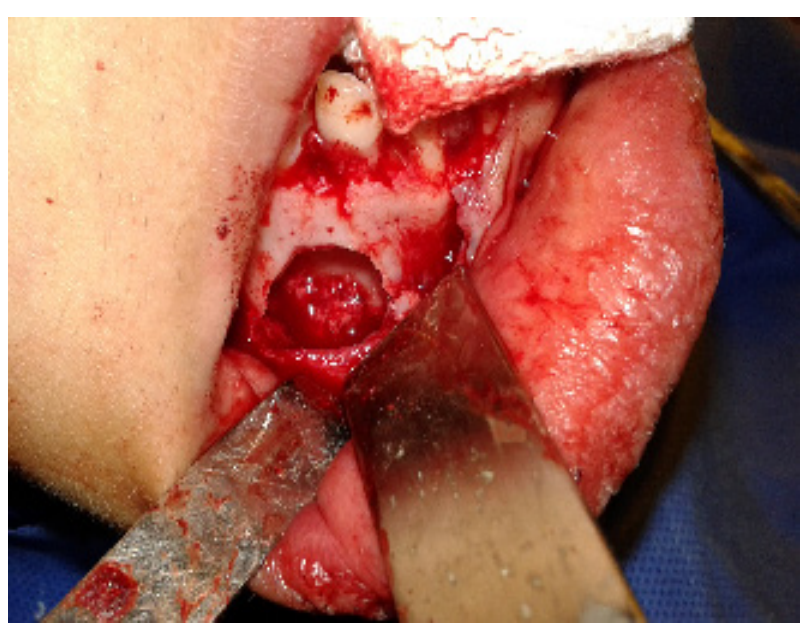

Figura 8A. Lecho quirúrgico. 


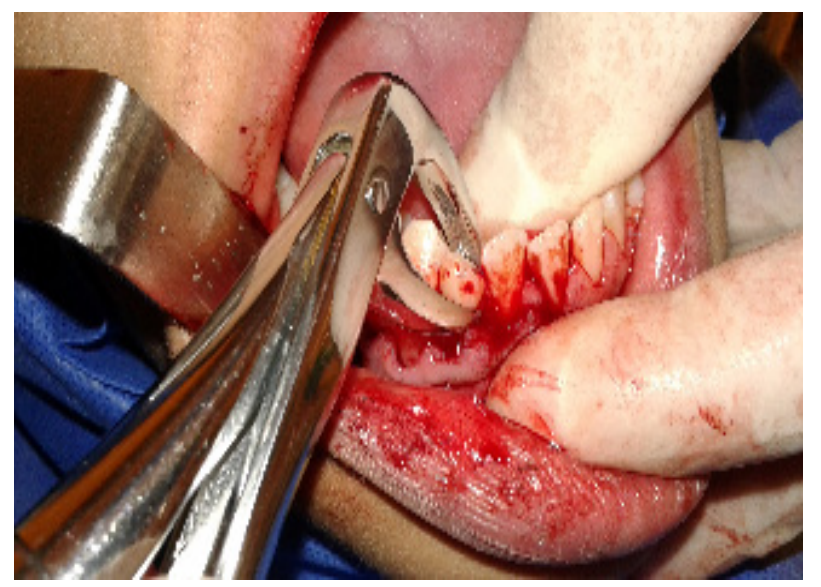

Figura 8B. Exodoncia de pieza 8.3.

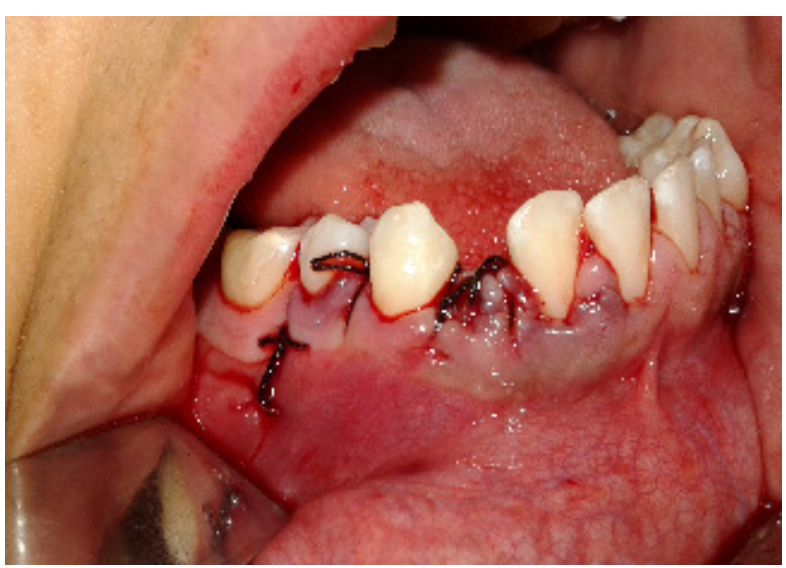

Figura 8C. Sutura.

diente) y tejidos blandos (membrana), según protocolo, se envía al Laboratorio de Patología Quirúrgica de la Facultad de Odontología de la Universidad de Buenos Aires (Figura 9).

A los 7 días se realiza el primer control posquirúrgico. Se retiran los puntos de sutura y se deriva al servicio de Endodoncia para realizar prueba de sensibilidad del incisivo lateral inferior derecho (4.2) por su proximidad con la lesión, dando como respuesta sensibilidad positiva en dicha pieza.

El diagnóstico anatomopatológico da como resultado: "Cuadro histopatológico

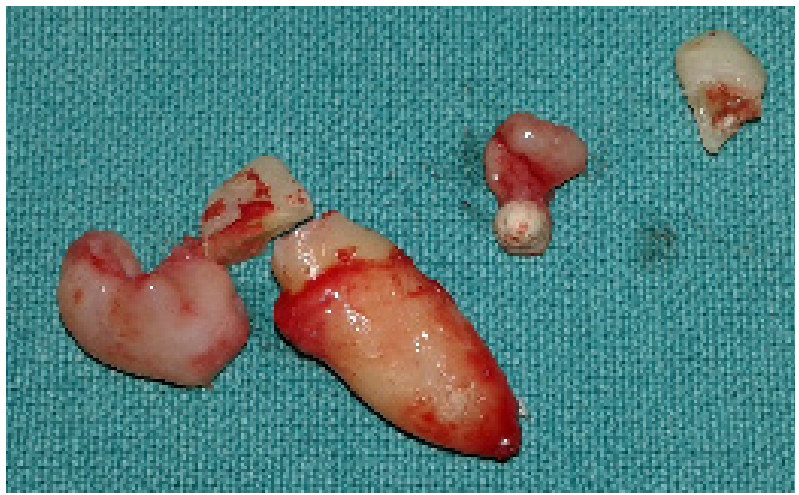

Figura 9. Pieza 4.3 con odontosección y dentículos extraídos.

correspondiente a un tumor odontogénico del tipo de los odontomas compatible con fibro - odontoma ameloblástico".

Los controles posquirúrgicos clínico radiográficos se realizan al mes, a los 3 y a los 6 meses de la intervención. En los mismos se observan las mucosas normales sin patologías asociadas y regeneración ósea acorde al tiempo posquirúrgico trascurrido.

Acorde al plan de tratamiento realizado, el paciente continúa su atención en el servicio de Odontología Preventiva e ingresa para tratamiento en el servicio de Ortodoncia, trabajando siempre en interrelación con los distintos servicios (Figura 10 y 11).

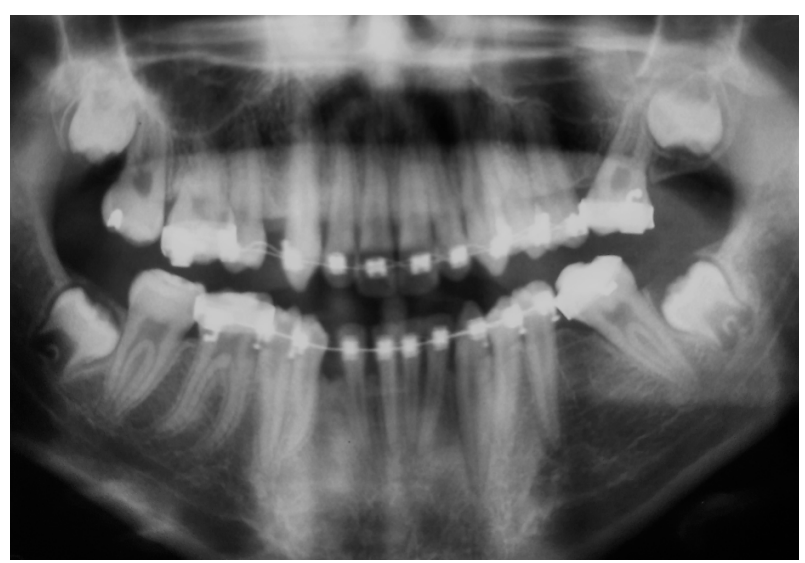

Figura 10. Radiografía panorámica posquirúrgica. Control a los 2 años. 


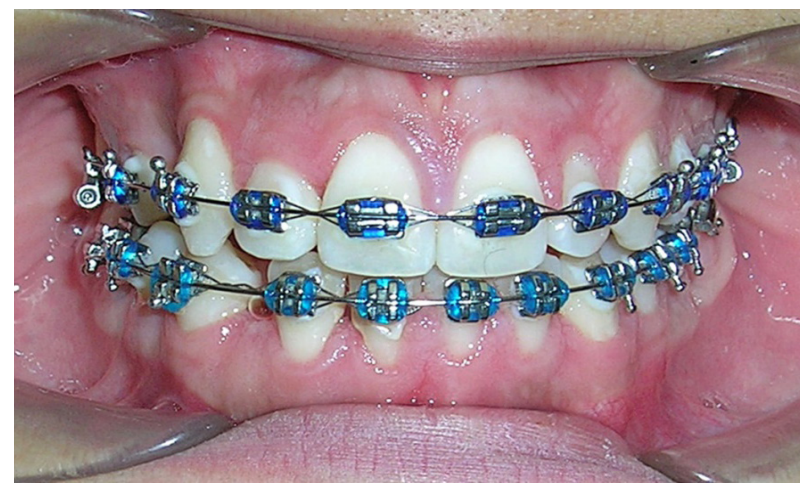

Figura 11. Aspecto intrabucal actual.

\section{Discusión}

La OMS en el año 2005 establece que el FOA consiste en una neoplasia benigna compuesta por epitelio odontogénico proliferativo, incluido en un tejido ectomesenquimático odontogénico celular similar a la papila dental, con diversos grados de cambios inductivos y formación de tejidos duros dentales. ${ }^{1,7}$ Otros autores directamente lo asocian a una etapa inmadura del odontoma complejo. ${ }^{7,16}$

Pese a lo anterior, el FOA no debe ser considerado un odontoma, debido a reportes de casos que muestran comportamientos neoplásicos tales como recurrencia y transformación maligna, aun habiendo realizado una cuidadosa excisión de la lesión incluyendo dientes no erupcionados desplazados. ${ }^{17}$

En este caso, el diagnóstico de la lesión es realizado por la persistencia clínica del canino inferior derecho temporario, que complementado con una radiografía periapical de la zona nos permite observar la retención de la pieza 4.3 y la presencia del FOA. Esto nos confirma la importancia de realizar el estudio radiográfico previo a toda extracción dentaria.
Como estudio radiográfico complementario, en el caso clínico presentado, se utiliza la técnica de Clark, ya que en ese momento nuestra institución no contaba con un tomógrafo para ver la ubicación de la pieza dentaria retenida y definir la zona de abordaje quirúrgico de la lesión. En la actualidad, la posibilidad de derivación a otro hospital público para la realización de un estudio tomográfico nos permite definir con certeza la ubicación de las piezas a tratar y su relación con los tejidos vecinos.

Algunos estudios indican que el FOA puede comportarse agresivamente con múltiples recurrencias. ${ }^{18,19}$ Se sugirió que un FOA principalmente benigno puede sufrir una transformación maligna en fibrosarcoma ameloblástico. Por lo tanto, es importante continuar el seguimiento de las lesiones de FOA durante varios años para garantizar que no haya signos futuros de recurrencia agresiva. $^{20}$

Este caso todavía se encuentra en observación y con controles clínicos y radiográficos. Los hallazgos que evidencian neoformación ósea en la región de la intervención, permiten concluir que la técnica quirúrgica elegida mostró ser segura y eficaz. Los controles radiográficos posoperatorios son imprescindibles, así como también el trabajo dentro de un equipo interdisciplinario para continuar con el plan de tratamiento indicado.

\section{Conclusiones}

La incidencia de tumores odontogénicos derivados del folículo dental, es más alta en la infancia y la mayoría cursa sin signos ni síntomas clínicos. El diagnóstico clínico 
y radiográfico precoz junto con el enfoque interdisciplinario, son fundamentales para realizar una derivación oportuna y el tratamiento temprano de pacientes en crecimiento, evitando así secuelas tardías.

En este caso clínico, el tratamiento radical realizado a partir de la extirpación completa de la lesión y extracción de la pieza dentaria retenida, corroboran la ausencia de recidiva o malignización de la lesión, evidenciado esto con los controles clínicos y radiográficos a distancia.
Este trabajo es avalado por el Comité de Docencia e Investigación y el Comité de Ética en Investigación del Hospital de Odontología Infantil "Don Benito Quinquela Martín", Ministerio de Salud - Gobierno de la Ciudad Autónoma de Buenos Aires, Argentina.

Los autores no presentan conflictos de interés ni fuentes de financiamiento en la realización de este artículo.

\section{Referencias bibliográficas}

1. Barnes L, Eveson JW, Reichart P, Sidransky D. World Health Organization Classification of tumours. IARC Press: Lyon 2005; 309. Disponible en: https://screening.iarc.fr/doc/BB9.pdf

2. Sapp P, Eversole L, Wysocky G. Patología Oral y Maxilofacial Contemporánea. Harcourt International 1998; 149-50.

3. Neville BW, Damm DD, Allen CM, Bouquot JE. Oral \& Maxillofacial Pathology $2^{\mathrm{a}}$ ed. St Louis: Saunders Elsevier; 2002; 628-9.

4. Correa F, Rebelo H, Sá J, Vieira S, Nogueira J, Paiva F, et al. Ameloblastic fibro-odontoma: case report with maintenance of the involved teeth. Int J Pediatr. Otorhinolaryngol Extra 2008; 3: 48-52. Disponible en: https://www.sciencedirect.com/science/article/abs/pii/S1871404807000792

5. Reichar PA, Philipsen Hp. Odontogenic tumors and allied lesions. Quintessence Publishing Co Ltd. $1^{\mathrm{a}}$ ed, London 2004.

6. Kumar S, Suvy M y cols. Ameloblastic fibro-odontoma. International Journal of Surgery Case Reports 2014; 5: 1142-4. Disponible en: https://www.ncbi.nlm.nih.gov/pmc/articles/PMC4276268/

7. Álvarez F, Licéaga R. Fibro - odontoma ameloblástico: Revisión de la literatura y presentación de un caso. Revista Odontológica Mexicana 2009; 13: 122 - 5. Disponible en: https://www.medigraphic.com/pdfs/ odon/uo-2009/uo092i.pdf

8. Cordero - Loria AV, Solano-Bonilla L. Fibro odontoma ameloblástico asociado a canino inferior derecho impactado. Rev Cient Odontol. 2015; 11: 30-3. Disponible en: https://www.redalyc.org/ pdf/3242/324242282005.pdf

9. Castellón L, Fariña R, Martínez B, Canto L. Fibro - odontoma ameloblástico de la mandíbula. Rev Esp Cir Oral Maxilofac.; 2013; 87-92. Disponible en: http://scielo.isciii.es/pdf/maxi/v35n2/caso4.pdf

10. Botero N, León R, Cantillo O, Álvarez P, Díaz A. Fibroma ameloblástico asociado a un odontoma compuesto. La importancia del estudio histopatológico. Rev Esp Cir Oral Maxilofac 2013; 175 - 80. Disponible en: http://scielo.isciii.es/pdf/maxi/v35n4/casoclinico04.pdf

11. Sreenath G, Indira Reddy Y, Sreenivasreddy P, Ravi Prakash A. Ameloblastic fibro - odontome (AFO) of the mandible: a case report. Journal of Clinical and Diagnostic Research 2014; 260 - 2. Disponible en: https:// www.ncbi.nlm.nih.gov/pmc/articles/PMC3939569/

12. Suarez - Portilla JC, Cantillo - Payares O, Urbano S, Harris - Ricardo J. Ameloblastic fibro-odontoma: conservative management with preservation of dental structures. Rev CSV 2019; 11: 143-50.

13. Reyes P, Álvarez C y cols. Report of a case of Fibrous Ameloblastic Odontoma. Rev Esp Cir Oral Maxilofac. 2009; 31: 337-42 
14. Praveena R. Ameloblastic fibro-odontoma in a kid - an impostor in radiography. Indian Journal of Research. 2017; 6: 14 - 6. Disponible en: https://www.researchgate.net/publication/343536972_AMELOBLASTIC_ FIBRO-ODONTOMA_IN_A_KID_-AN_IMPOSTOR_IN_RADIOGRAPHY_PARIPEX_-INDIAN_JOURNAL_ OF_RESEARCH

15. Biondi AM, Cortese S. Odontopediatría. Fundamentos y prácticas para la atención integral personalizada. Alfaomega. Buenos Aires.2010; 4: 29-51

16. Dolanmaz D, Pampu A, Kalayci A, Etöz O, Atici S. An unusual size of ameloblastic fibro - odontoma. Dentomaxillofac Radiol. 2008; 37: 179 - 82.

17. Verdugo M, Hidalgo A, Venegas B, Plana S, Schilling A. Ameloblastic fibro odontoma in the mandible: a case report and review of the literature. Anuario Sociedad de Radiología Oral Máxilo Facial de Chile 2014; 17 : 40 - 6. Disponible en:http://sociedadradiologiaoral.cl/doc/anuarios_div/2014/anuario2014-42-48.pdf

18. Chen Y, Li TJ, Gao Y, Yu SF. Fibroma ameloblástico y lesiones relacionadas: un estudio clínico-patológico con referencia a su naturaleza e interrelación. J Oral Pathol Med 2005; 34: 588-95.

19. Delgado - Azañero W, Funes - Rumiche I, Torres - Vega F, Calderón - Ubaqui V. Fibrosarcoma ameloblástico encapsulado. Rev Estomatol Herediana. 2008;18 :128-35.Disponible en: https://www.researchgate.net/ publication/299402705_Fibrosarcoma_ameloblastico_encapsulado

20. Kirjavainen A, Tuovinen V, Sándor GK. Gran fibroodontoma ameloblástico en una niña de 7 años con análisis de 108 casos. Ann Maxillofac Surg 2016; 6: 15-20.

Recibido: 16/03/2021

Aceptado: 08/07/2021

Correspondencia: Graciela Susana Martínez, correo: gracismartinez@yahoo.com.ar 